\title{
Metformin attenuates hypoxia-induced resistance to cisplatin in the HepG2 cell line
}

\author{
HIROMASA FUJTAA $^{1}$, KATSUMI HIROSE ${ }^{1,2}$, MARIKO SATO $^{1}$, \\ ICHITARO FUJIOKA $^{1}$, TAMAKI FUJITA ${ }^{1}$, MASAHIKO AOKI $^{1 *}$ and YOSHIHIRO TAKAI $^{1,2^{*}}$ \\ ${ }^{1}$ Department of Radiology and Radiation Oncology, Hirosaki University Graduate School of Medicine, Hirosaki, \\ Aomori 036-8562; ${ }^{2}$ Department of Radiation Oncology, Southern Tohoku BNCT Research Center, \\ Koriyama, Fukushima 963-8052, Japan
}

Received March 14, 2018; Accepted November 14, 2018

DOI: $10.3892 / \mathrm{ol} .2018 .9869$

\begin{abstract}
Hepatoblastoma is the most commonly occurring liver tumor in children. Preoperative chemotherapy and surgery have improved treatment outcomes; however, further improvements are required in the treatment of advanced cases. Recently, the efficacy of transarterial chemoembolization (TACE) has garnered attention. TACE increases the local concentration of drugs by transcatheterically administering antitumor agents, and induces necrosis in the tumor by embolizing the feeding artery. However, studies have revealed that tumors exhibit resistance to anticancer drugs in hypoxic environments. Metformin is a drug used to treat type 2 diabetes; however, recent reports have indicated that it may also exhibit antitumor effects in various cancer cell lines. These effects are hypothesized to be mediated by the activation of adenosine monophosphate-activated protein kinase and reduction of mammalian target of rapamycin signaling, but these effects occur at high concentrations that are not suitable for use in a clinical setting. The potential efficacy of metformin at increased physiological concentrations has not been evaluated. The present study investigated the therapeutic effect of low concentrations of metformin in combination with cisplatin on liver cancer HepG2 cells in hypoxic conditions. HepG2 cells were treated with cisplatin alone, metformin alone, or a combination of these two drugs and cultured in normoxia or hypoxia. Treatment with either $5 \mu \mathrm{M}$ cisplatin or $1 \mathrm{mM}$ metformin alone did not significantly affect cell proliferation or apoptosis in hypoxic conditions. However, when $5 \mu \mathrm{M}$ cisplatin was combined with $1 \mathrm{mM}$
\end{abstract}

Correspondence to: $\mathrm{Dr}$ Katsumi Hirose, Department of Radiation Oncology, Southern Tohoku BNCT Research Center, 7-10 Yatsuyamada, Koriyama, Fukushima 963-8052, Japan

E-mail: khirose@hirosaki-u.ac.jp

${ }^{*}$ Contributed equally

Key words: hepatoblastoma, liver cancer, HepG2 cell, transarterial chemoembolization, metformin, cisplatin, hypoxia metformin, a significant inhibition of cell proliferation and induction of apoptosis was observed in hypoxic HepG2 cells. In conclusion, a low concentration of metformin attenuates hypoxia-induced resistance to cisplatin in HepG2 cells. Selective delivery of an effective dose of metformin to a hepatoblastoma tumor may be achievable and clinically useful with TACE.

\section{Introduction}

The most common type of childhood liver tumor is hepatoblastoma, which is a rare tumor that usually affects children younger than 3 years of age (1). The treatments for hepatoblastoma are surgery and chemotherapy (2). Although surgery is a definitive treatment for this tumor, cisplatin-based preoperative chemotherapy has improved treatment outcomes (3). However, in high-risk groups, such as those with metastasis, tumors in all liver segments, abdominal extrahepatic disease, major vascular invasion, low $\alpha$ fetoprotein, or tumor rupture, outcomes remain poor, and more effective therapies are needed (4). Transarterial chemoembolization (TACE) has been used for the treatment of patients with various malignant tumors with some success (5). Because anticancer agents are administered through the artery that feeds the tumor, drugs can reach the tumor at high concentration, and systemic side effects are reduced (6). In recent years, several reports have indicated that TACE as a neoadjuvant therapy improves the resection rate of otherwise unresectable hepatoblastoma compared with systemic neoadjuvant chemotherapy (7). However, occlusion of the feeding artery with embolic materials inevitably results in partial hypoxic conditions in the tumor microenvironment, as well as tumor necrosis (8). Previous studies have reported that hypoxic conditions play a role in acquired resistance to chemotherapy and radiotherapy. Furthermore, some evidence suggests that hypoxia is involved in tumor metastasis and recurrence (9), and hypoxia induced by an embolus may inhibit the efficacy of chemotherapy. Therefore, reduction of hypoxia may be a strategy for cancer therapy, especially in combination with TACE treatment.

Metformin is a drug used for the treatment of type 2 diabetes and has recently been shown to have antitumor effects on some 
cancer cell lines (10-17). Metformin preferentially targets cancer stem cell-like cells that may be the key drivers of tumor recurrence in hypoxic conditions $(15,18,19)$. However, regarding liver tumors, metformin is cytotoxic for HepG2 cells only at concentrations over $10 \mathrm{mM}(20)$, which is about $2 \times 10^{3}$ times higher than the concentration reached in human plasma after twice daily oral administration of a 500-mg clinical dose of metformin (21). Systemically, such a high concentration may affect normal tissue cells with resulting adverse lactate acidosis and potentially other serious side effects. Assuming that selective delivery of metformin to the tumor at an effective dose can be achieved by TACE, we aimed to investigate whether metformin can modulate acquired chemoresistance following partial tumor hypoxia, which may be induced by TACE in clinical application.

\section{Materials and methods}

Materials. Dulbecco's modified Eagle's medium (DMEM), RPMI, and fetal bovine serum (FBS) were obtained from Sigma-Aldrich; Merck KGaA (Darmstadt, Germany). Penicillin and streptomycin were obtained from Gibco; Thermo Fisher Scientific, Inc. (Waltham, MA, USA). Cisplatin was obtained from Nichi-Iko Pharmaceutical Co. (Toyama, Japan). Metformin was obtained from Tokyo Chemical Industry Co. (Tokyo, Japan).

Cell culture and growth conditions. The human liver cancer cell line HepG2, the human lung cancer cell line A549, and the human oral squamous carcinoma cell line SAS were purchased from the Riken BioResource Center (Tsukuba, Ibaraki, Japan). The human head and neck squamous cell carcinoma line CA9-22, HSC4 and HO-1-u-1 cells were purchased from the Cell Resource Center for Biomedical Research, Institute of Development, Aging and Cancer, Tohoku University (Sendai, Miyagi, Japan). The human hepatoblastoma cell line HuH-6 were purchased from the Japanese Collection of Research Bioresources Cell Bank (Ibaraki, Osaka, Japan). HepG2 cells, A549 and HuH-6 cells were grown in DMEM and SAS, CA9-22, HSC4 and HO-1-u-1 cells were grown in RPMI. Both types of media were supplemented with penicillin, streptomycin, and $10 \%$ heat-inactivated FBS, and cells were grown at $37^{\circ} \mathrm{C}$ in a humidified atmosphere containing $5 \% \mathrm{CO}_{2}$. Hypoxia was defined as $1 \%$ oxygen, which was achieved by culturing cells in modular incubator chambers (Billups-Rothenberg, Del Mar, CA, USA) that were flushed with gas mixtures (95\% nitrogen $/ 5 \%$ carbon dioxide) and sealed to maintain hypoxia after checking oxygen concentrations with an oxygen monitor (JKO-02 Ver. III; JIKCO, Tokyo, Japan).

Cell viability analysis. HepG2 cells were incubated in 24-well ELISA plates (Iwaki, Chiba, Japan) in $1 \mathrm{ml}$ culture medium at a density of $5 \times 10^{5}$ cells. A range of concentrations was assessed for cisplatin $(0-25 \mu \mathrm{M})$ and metformin (0-5 mM). For the trypan blue dye exclusion test, cells were stained using phosphate-buffered saline (PBS) containing $0.1 \%$ trypan blue (Nacalai Tesque, Inc., Kyoto, Japan). Cell viability was assessed by counting the number of unstained cells using the TC20 ${ }^{\mathrm{TM}}$ automated cell counter (Bio- Rad Laboratories, Inc., Hercules, CA, USA). Also, SAS, HO-1-u-1,
HSC4 A549 and HuH-6 cells were incubated in 24-well ELISA plates (Iwaki) in $1 \mathrm{ml}$ culture medium at a density of $1.5-5 \times 10^{5}$ cells depending on the appropriate condition for confluence. A range of concentrations was assessed for cisplatin $(0$ or $5 \mu \mathrm{M})$ and metformin $(0$ or $1 \mathrm{mM})$. The trypan blue assay was performed as described above.

Cell cycle analysis. The cell cycle phase distribution was analyzed using propidium iodide (PI)-RNase (Immunostep, Salamanca, Spain) staining according to the manufacturer's instructions. Briefly, cells were harvested following a 24-h incubation in 6-cm culture dishes (Iwaki) in each condition. After washing with binding buffer, cells were re-suspended in the buffer with PI-RNase. Stained cells were analyzed for PI fluorescence using Cytomics FC 500 (Beckman Coulter, Inc., Brea, CA, USA).

Detection of apoptosis. Apoptotic cells were detected using Annexin V-fluorescein isothiocyanate (FITC) and 7-Aminoactinomycin D (7-AAD; BD Biosciences, San Jose, CA, USA) according to the manufacturer's instructions. Briefly, after a 12-h incubation in 24-well ELISA plates in each condition, cells were harvested, washed, suspended in $100 \mu \mathrm{l}$ binding buffer, and stained with Annexin V-FITC and 7-AAD for $15 \mathrm{~min}$ at room temperature in the dark. Apoptotic cells were determined using Cytoflex (Beckman Coulter, Inc.). After 7AAD-positive cells were excluded from gating, the fraction of Annexin V-positive cells was evaluated. FlowJo (Tomy Digital Biology Co., Ltd.) software was used to analyze the cytometric data.

Western blot analysis. HepG2 cells were incubated in 6-cm culture dishes (Iwaki) at a density of $1 \times 10^{6}$ cells. After a 12-h incubation, cells were treated with cisplatin and metformin in hypoxia for 6,12 , or $24 \mathrm{~h}$. Cells were lysed using Cell Lysis Buffer (cat. no. 9803s; Cell Signaling Technology, Inc., Danvers, MA, USA) and protease inhibitor cocktail (cat. no. 635673; Takara Bio Inc., Shiga, Japan). Cell lysates and pre-stained molecular weight markers were separated by SDS-PAGE with $10 \%$ Mini-PROTEAN ${ }^{\circledR}$ TGX $^{\mathrm{TM}}$ precast gels, followed by transfer onto polyvinylidene fluoride membranes with Trans-Blot ${ }^{\circledR}$ Turbo $^{\mathrm{TM}}$ (both Bio-Rad Laboratories, Inc.). The membranes were blocked with Block $\mathrm{ACE}^{\circledR}$ (cat. no. UK-B80; DS Pharma Biomedical Co., Ltd., Osaka, Japan) dissolved in distilled $\mathrm{H}_{2} \mathrm{O}$ and then incubated with various primary antibodies diluted in blocking buffer, Can Get Signal ${ }^{\circledR}$ solution 1 (Can Get Signal ${ }^{\circledR}$ Immunoreaction Enhancer Solution; Toyobo Biochemicals, Osaka, Japan). Rabbit monoclonal anti-p-adenosine monophosphate-activated protein kinase (AMPK) $\alpha$ antibody (cat. no. 2535; $1: 1,000)$, rabbit polyclonal anti-p-AMPK $\beta 1$ antibody (cat. no. 4186; 1:1,000), rabbit anti-p-Akt (Ser473) antibody (cat. no. 4046; 1:1,000), rabbit monoclonal anti-p-mammalian target of rapamycin (mTOR) (Ser2448) antibody (cat. no. 5536; 1:1,000; all Cell Signaling Technology, Inc.), mouse monoclonal anti-hypoxia-induced factor $1 \alpha(\mathrm{HIF}-1 \alpha)$ antibody (cat. no. 610959; 1:500; BD Biosciences), and mouse monoclonal anti-actin antibody (cat. no. sc-47778; 1:1,000; Santa Cruz Biotechnology, Dallas, TX, USA) were incubated for $1 \mathrm{~h}$. The blots were then washed three 
times with Tris-buffered saline containing $0.1 \%$ Tween-20 (Bio-Rad Laboratories, Inc.) and incubated with anti-mouse IgG, horseradish peroxidase-linked antibody (cat. no. 7076; 1:2,000; Cell Signaling Technology, Inc.) in blocking buffer Can Get Signal ${ }^{\circledR}$ solution 2 (Can Get Signal ${ }^{\circledR}$ Immunoreaction Enhancer Solution; Toyobo Biochemicals) for $1 \mathrm{~h}$. Membranes were washed three times, and immunoreactivity was visualized with Clarity Western ECL substrate using a chemiluminescence Molecular Imager ${ }^{\circledR}$ ChemiDoc $^{\mathrm{TM}}$ XRS+ system (both Bio-Rad Laboratories, Inc.) according to the manufacturer's instructions.

Transfection with small interfering RNA (siRNA). Silencing of HIF-1 $\alpha$ gene expression was achieved by the siRNA strategy. HepG2 cells were incubated in 96-well ELISA plates at a density of $2 \times 10^{4}$ cells. Cells were transfected with siRNA targeting human HIF1A (MISSION ${ }^{\circledR}$ SiRNA; Sigma-Aldrich; Merck KGaA) using the TransIT ${ }^{\circledR}$-mRNA Transfection kit (Takara Bio Inc.). BLOCK-iT ${ }^{\mathrm{TM}}$ Alexa Fluor $^{\mathrm{TM}}$ Red Fluorescent Control (Invitrogen; Thermo Fisher Scientific, Inc.) was used as a control. The inhibitory effect of siRNA on HIF1A gene expression was confirmed using quantitative real-time reverse transcription polymerase chain reaction (PCR) with the calibration curve method.

Total RNA extraction and reverse transcription-quantitative PCR (RT-qPCR). Total RNA was isolated using Agencourt ${ }^{\circledR}$ RNAdvanced Tissue ${ }^{\mathrm{TM}}$ (Beckman Coulter, Inc.) and Agencourt's patented solid phase reversible immobilization (SPRI) paramagnetic bead technology, according to the manufacturer's instructions. Briefly, cultured cells were lysed with lysis buffer and proteinase $\mathrm{K}$ and transferred into new 96-multi-well plates. After total RNA was mixed with paramagnetic beads, the beads were washed with wash buffer and $70 \%$ ethanol, and separated from contaminants using Agencourt SPRIPlate 96R. Subsequently, DNase I solution (Thermo Fisher Scientific, Inc.) was added to each well to digest the genomic DNA. Total RNA was eluted from the magnetic particles with nuclease-free water (Thermo Fisher Scientific, Inc.). Then, first-strand cDNA was synthesized from the isolated total RNA using iScript ${ }^{\mathrm{TM}}$ RT Supermix for RT-qPCR ${ }^{\circledR}$ (Bio-Rad Laboratories) according to the manufacturer's instructions. Gene expression was assessed using qRT-PCR with SsoAdvanced Universal SYBR-Green Supermix ${ }^{\circledR}$ (Bio-Rad Laboratories) with typical amplification parameters of $95^{\circ} \mathrm{C}$ for $30 \mathrm{sec}$, followed by 40 cycles at $98^{\circ} \mathrm{C}$ for $10 \mathrm{sec}$ and $60^{\circ} \mathrm{C}$ for $30 \mathrm{sec}$. Relative differences were determined by the crossing point method with a standard curve. The mRNA expression in each condition was compared after normalization to expression of the housekeeping gene GAPDH. The oligonucleotide primer sets used for real-time PCR purchased from Takara Bio Inc. were as follows: GAPDH forward, 5'-GCACCGTCAAGGCT GAGAAC-3' and reverse, 5'-TGGTGAAGACGCCAGTGG A-3'; HIF1A forward, 5'-CTCATCAGTTGCCACTTCCAC ATA-3' and reverse, 5'-AGCAATTCATCTGTGCTTTC ATGTC-3'.

Measurement of mitochondrial $\mathrm{O}_{2}{ }^{-}$. The MitoSOX ${ }^{\mathrm{Tm}}$ RED Mitochondrial $\mathrm{O}_{2}{ }^{--}$Indicator (Invitrogen; Thermo Fisher
Scientific, Inc.) was used to detect mitochondrial $\mathrm{O}_{2}{ }^{--}$. Briefly, cells were incubated with $5 \mu \mathrm{M}$ cisplatin and $1 \mathrm{mM}$ metformin in hypoxia, washed in PBS, and incubated with $5 \mu \mathrm{M}$ MitoSOX ${ }^{\mathrm{TM}} \mathrm{RED}$ at $37^{\circ} \mathrm{C}$ for $30 \mathrm{~min}$ according to the manufacturer's instructions. The cells were then washed, re-suspended in $300 \mu \mathrm{l}$ PBS, and analyzed using Cytomics FC 500 (Beckman Coulter, Inc.).

HepG2 cells were incubated in 24-well ELISA plates (Iwaki). After a 24-h incubation, cells were treated with cisplatin $(0$ or $5 \mu \mathrm{M})$, metformin $(0$ or $1 \mathrm{mM})$ and $\mathrm{N}$-acetyl-1-cysteine (NAC; 0 or $10 \mathrm{mM}$ ) in hypoxic conditions in $48 \mathrm{~h}$. The effect of NAC was evaluated by MitoSOX ${ }^{\mathrm{TM}}$ RED and the trypan blue assay performed as described above.

Statistical analysis. Results are presented as the mean \pm standard error of the mean. Significant differences were determined using the two-sided Student's t-test or Welch's t-test for unpaired two group, depending on the data distribution. One-way analysis of variance (ANOVA) or two-way ANOVA were used for multiple comparisons, followed by Turkey-Kramer's test or Dunnett's test. $\mathrm{P}<0.05$ was considered to indicate a statistically significant difference. Excel 2013 software (Microsoft Corp., Redmond, WA, USA), with the add-in software Statcel 4 (OMS Publishing Inc., Tokorozawa, Saitama, Japan, http://www.oms-publ.co.jp/), was used for statistical analysis.

\section{Results}

Influence of hypoxic conditions on cell growth and viability. We first investigated the cytotoxic effects of hypoxic conditions in HepG2 cells. The cells were incubated for 0, 12, 24, or $48 \mathrm{~h}$ in normoxic or hypoxic $\left(1 \% \mathrm{O}_{2}\right)$ conditions, and then trypan blue dye exclusion tests and cell cycle analyses were performed. The number of viable HepG2 cells following incubation in normoxia significantly increased over time. In hypoxic conditions, the cell growth rate was almost the same as in normoxia up to $12 \mathrm{~h}$. However, the proliferation rate decreased after $12 \mathrm{~h}$, and the number of cells remained at the same level from 24 to $48 \mathrm{~h}$. The number of viable HepG2 cells was significantly different between normoxic and hypoxic conditions at $48 \mathrm{~h}(\mathrm{P}=0.004$; Fig. 1A).

The proportion of cells in G1 at 24 h significantly increased in hypoxic conditions compared with normoxic conditions $(\mathrm{P}<0.01)$. In addition, the proportion of cells in $\mathrm{S}$ or $\mathrm{G} 2 / \mathrm{M}$ at $24 \mathrm{~h}$ significantly decreased in hypoxic conditions compared with normoxia $(\mathrm{P}<0.01)$. Cells showed $\mathrm{G} 1$ arrest in hypoxic conditions (Fig. 1B).

Impact of oxygen status on cytotoxic effect of cisplatin. To determine the effects of cisplatin on HepG2 cell viability, HepG 2 cells were treated with different concentrations of cisplatin for $48 \mathrm{~h}$ in normoxic or hypoxic conditions. In hypoxia, the cytotoxic effect of cisplatin was attenuated compared to normoxia, and the effect was significant at $5 \mu \mathrm{M}$ or more (Fig. 2).

Next, we used flow cytometry to investigate the effect of cisplatin $(0-5 \mu \mathrm{M})$ on induction of apoptosis. In normoxic conditions, Annexin V-FITC-positive apoptotic cells increased depending on the concentration of cisplatin. 

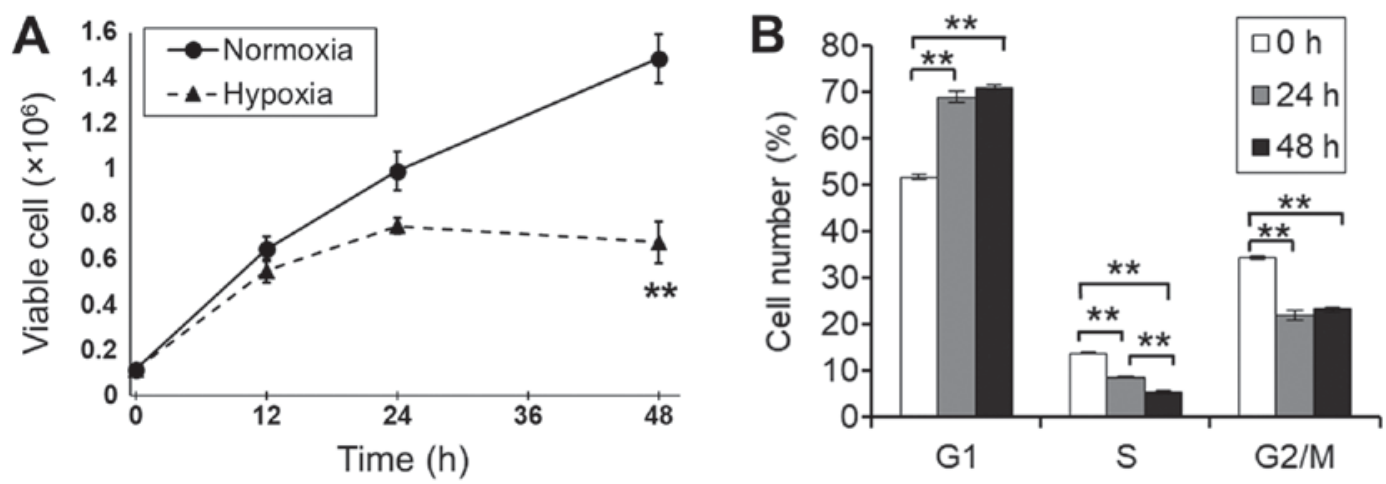

Figure 1. The influence of hypoxia on survival and cell growth in the HepG2 liver cancer cell line. (A) HepG2 cells showed time-dependent growth in normoxic conditions, but proliferation was significantly inhibited in hypoxic conditions, with a significant difference at $48 \mathrm{~h}$ compared to normoxia $(\mathrm{P}=0.005)$. (B) HepG2 cells showed G1 arrest in hypoxic conditions. The results represent the mean \pm SE of three independent experiments; * $\mathrm{P}<0.01$ vs. normoxic conditions, as determined by Student's t-test for each time point (A), or the cell cycle distribution at $0 \mathrm{~h}$, as determined by one-way ANOVA with Turkey-Kramer's post-hoc test (B). ANOVA, one-way analysis of variance.

However, in hypoxic conditions, no significant change was observed (Fig. 3A). In normoxic conditions, the number of apoptotic cells at $24 \mathrm{~h}$ increased at a concentration of $5 \mu \mathrm{M}$ $(\mathrm{P}<0.05)$ and at $48 \mathrm{~h}$ increased at concentrations of 1 or $5 \mu \mathrm{M}(\mathrm{P}<0.01)$. In the hypoxic conditions, cisplatin induced apoptosis at $24 \mathrm{~h}(\mathrm{P}<0.05)$ but did not induce it at $48 \mathrm{~h}$ (Fig. 3B and C). Necrotic cell were no significant change in each conditions.

Effects of metformin on HepG2 cell viability and apoptosis in hypoxic conditions. We observed that metformin inhibited the proliferation of HepG2 cells in both normoxia and hypoxia, with a concentration of $5 \mathrm{mM}$ significantly reducing cell viability in each condition $(\mathrm{P}<0.01$; Fig. $4 \mathrm{~A})$. Next, we investigated the effect of combined treatment with cisplatin and metformin. Cisplatin decreased viability in a concentration-dependent manner, but we observed no significant effect with or without metformin (Fig. 4B). Although cisplatin alone had no effect on cell proliferation in hypoxic conditions, in the presence of $1 \mathrm{mM}$ metformin, cisplatin demonstrated antitumor effects in hypoxia $(\mathrm{P}=0.024$ at $5 \mu \mathrm{M}$ and $\mathrm{P}=0.005$ at $10 \mu \mathrm{M}$; Fig. $4 \mathrm{C}$ ).

In normoxic conditions, cisplatin combined with metformin did not affect apoptosis compared with cisplatin alone (Fig. 4D). The combination treatment with these two agents in hypoxic conditions increased the number of apoptotic cells compared to treatment with cisplatin alone ( $\mathrm{P}=0.031$; Fig. 4E).

To confirm the potential clinical relevance of these effects, we also tested the effects on the other hepatoblastoma HuH-6 cell line and the other cancerous cell lines that are often treated with cisplatin in the clinic, such as a lung adenocarcinoma A549 cells, head and neck squamous cell carcinoma SAS, HO-1-u-1 and HSC4 cells. For SAS and HO-1-u-1 cells, the cisplatin-induced cytotoxicity was enhanced selectively by combining metformin in hypoxic cells that acquired resistance to cisplatin compared to normoxic cells ( $\mathrm{P}=0.04$ for SAS, and $\mathrm{P}=0.03$ for HO-1-u-1, respectively) (Fig. 5A-D). Also in A549 cells, there was a tendency of significance for these effects $(\mathrm{P}=0.064)$ On the other hand, for HuH-6 and HSC4 cells, no obvious resistance

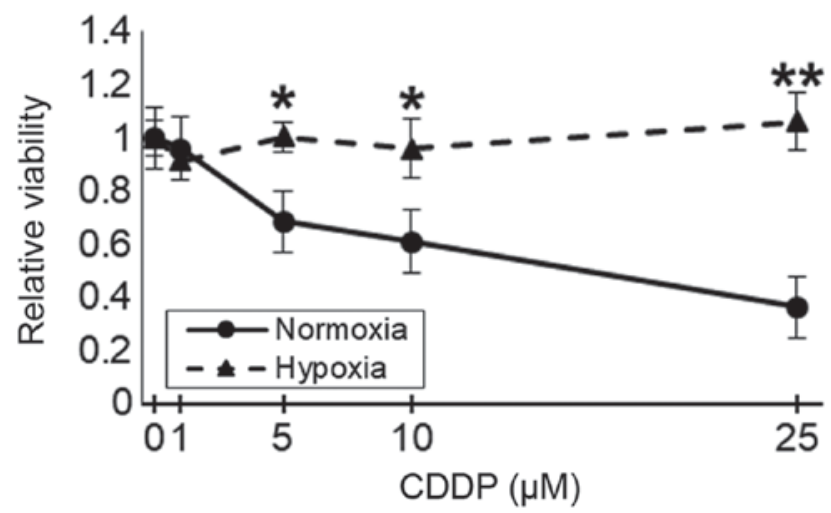

Figure 2. Impact of oxygen status on the cytotoxic effect of cisplatin. In hypoxia, the cytotoxicity of cisplatin was attenuated compared to normoxia, and this effect was significant at $5 \mu \mathrm{M}$ or more. The results represent the mean $\pm \mathrm{SE}$ of three independent experiments; ${ }^{*} \mathrm{P}<0.05$ and ${ }^{* *} \mathrm{P}<0.01$ vs. normoxic conditions, as determined by Student's t-test for each concentration. CDDP, cisplatin.

to cisplatin was induced by hypoxia, and no significant change was observed when metformin was added to these cells (Fig. 5E-J). These results suggest that the hypoxic cell-selective attenuation of the cytotoxicity of cisplatin by metformin may depend on the acquisition of treatment resistance induced by hypoxia, which is different for each cell type.

HIF-1a mediates a pathway in which metformin modulates hypoxia-induced resistance to cisplatin. Administration of metformin suppresses the respiratory chain in mitochondria and produces reactive oxygen species (ROS) in cells (22). Therefore, we first examined whether ROS is involved in the effect of metformin. HepG2 cells were cultured with $5 \mu \mathrm{M}$ cisplatin with or without metformin in hypoxic conditions. We observed no significant change in MitoSOX RED-positive cells between mock and metformin treatment $(19.7 \pm 1.6$ vs. $21.6 \pm 3.9 \%$, respectively; $\mathrm{P}=0.61)$ (Fig. $6 \mathrm{~A})$. NAC $(10 \mathrm{mM})$ induced a decrease in MitoSOX RED-positive cells in both mock and metformin-treated cells (14.5 \pm 1.2 , $15.9 \pm 4.1 \%$, respectively) (Fig. 6B). NAC did not reduce the 


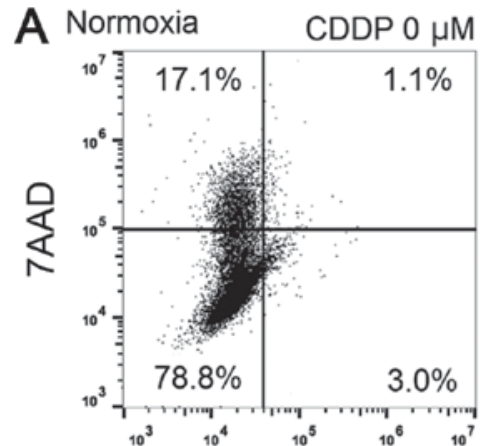

Annexin V-FITC

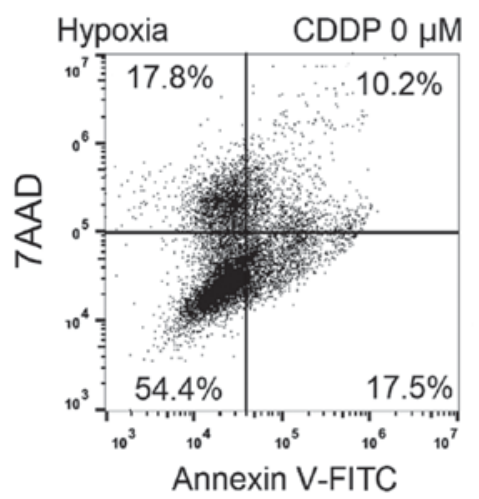

B

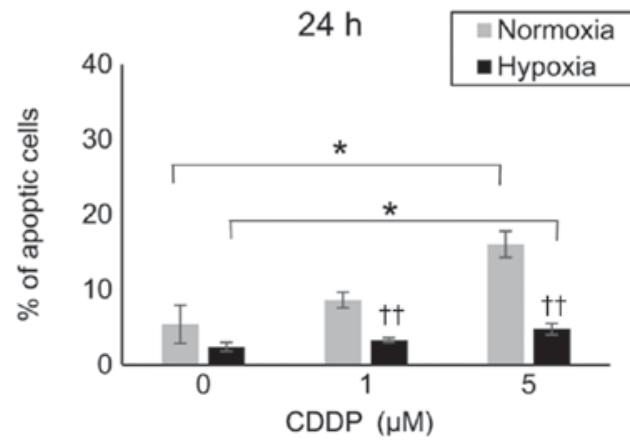

$1 \mu \mathrm{M}$

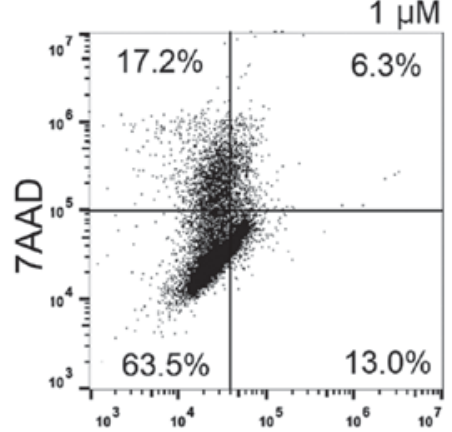

Annexin V-FITC

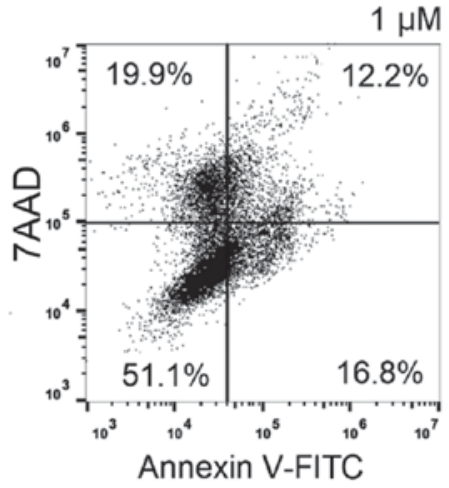

$1 \mu \mathrm{M}$
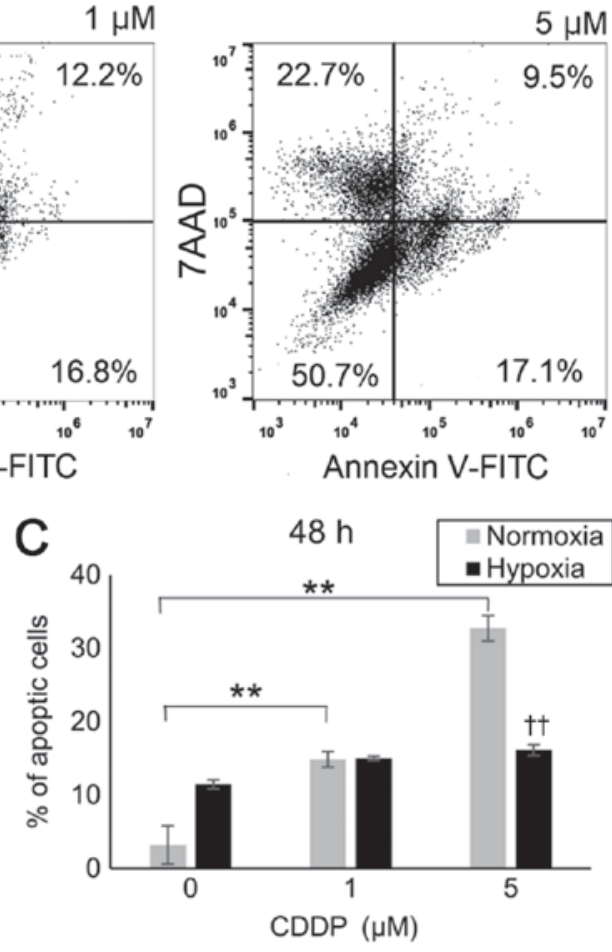

Figure 3. Hypoxia inhibits induction of apoptosis by cisplatin in HepG2 cells. Flow cytograms of cells harvested from cultures after treatment with cisplatin were analyzed. (A) Of the 7-AAD-negative population in these gated cells, a fraction of Annexin V-positive cells was induced by cisplatin in normoxic conditions. Apoptosis was induced by cisplatin in normoxic conditions at $48 \mathrm{~h}$, but the change was not clear in hypoxic conditions. (B and C) These values were expressed as a percentage. Induction of apoptosis by cisplatin increased in a dose-dependent manner in normoxic conditions $(\mathrm{P}<0.05$ at $5 \mu \mathrm{M}$ at $24 \mathrm{~h}$ and $\mathrm{P}<0.01$ at 1 and $5 \mu \mathrm{M}$ at $48 \mathrm{~h}$ ), but this effect was attenuated at $48 \mathrm{~h}$ in hypoxic conditions. The results represent the mean \pm SE of three independent experiments; ${ }^{*} \mathrm{P}<0.05$ and ${ }^{* *} \mathrm{P}<0.01$ vs. untreated cells for each oxygen condition, as determined by one-way ANOVA by following Dunnett's test. ${ }^{* T} \mathrm{P}<0.01$ vs. normoxic conditions, as determined by Student's t-test for each concentration. CDDP, cisplatin; FITC, Fluorescein isothiocyanate; 7-AAD, 7-Aminoactinomycin D.

number of viable cells (Fig. 6C). These results did not support the involvement of ROS in the effects of metformin.

In addition, metformin induces an antitumor effect via inhibition of mTOR by activation of AMPK (22). Therefore, we evaluated the expression and phosphorylation of a series of relevant proteins. HepG2 cells were cultured with $5 \mu \mathrm{M}$ cisplatin with or without metformin in hypoxic conditions. We observed no difference in the expression of $\mathrm{p}$-AMPK $\alpha$ or p-AMPK $\beta$ between the presence and absence of metformin. In untreated hypoxic cells, activation of Akt and mTOR was not observed, but activation of Akt and mTOR was enhanced by cisplatin administration. Administration of metformin suppressed the activity of Akt and mTOR. This change was remarkable at $24 \mathrm{~h}$. HIF-1 $\alpha$ was expressed in hypoxic conditions and was remarkably inhibited at $24 \mathrm{~h}$ after metformin administration (Fig. 7A).
To confirm the involvement of HIF-1 $\alpha$ in the mechanism of the effects of metformin, we also examined HIF-1 $\alpha$ knockdown cells following transfection of siRNA, which significantly inhibited HIF-1 $\alpha$ gene expression (Fig. 7B). The siRNA control and siHIF-1 $\alpha$ cells were treated with $5 \mu \mathrm{M}$ cisplatin with or without metformin in hypoxic conditions. For the sicontrol-treated cells, although there was assumed to be the tendency that the toxicity of cisplatin was enhanced with combining metformin $(\mathrm{P}=0.087$ in comparison of with or without combining metformin on Student's t-test), significant difference was not seen correctly on two-way ANOVA statistical analysis in this experimental condition $(\mathrm{P}=0.134)$. On the other hand, combining metformin had no effect in the siHIF-treated cells $(\mathrm{P}=0.35)$ (Fig. 7C and D).

These results suggested that HIF-1 $\alpha$ is involved in the modulation of resistance to cisplatin by metformin. In addition, 

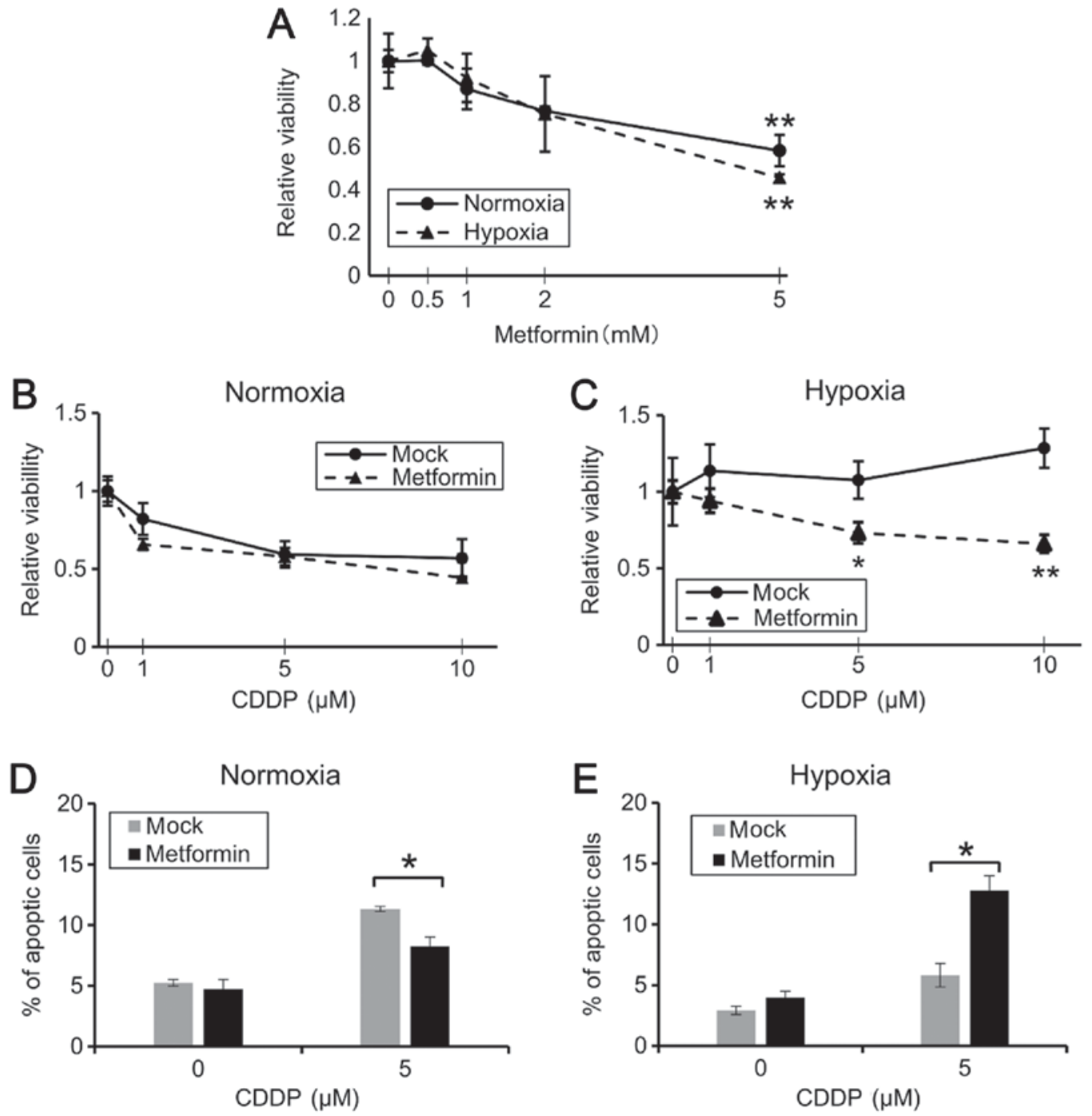

Figure 4. Attenuation of hypoxia-induced cisplatin resistance by a non-toxic concentration of metformin. (A) Metformin lower than $2 \mathrm{mM}$ did not inhibit the proliferation of HepG2 cells in either normoxic or hypoxic conditions. The results represent the mean $\pm \mathrm{SE}$ of three independent experiments as determined by one-way ANOVA by following Dunnett's test. (B) In normoxia, cisplatin showed the same cytotoxicity regardless of the presence or absence of metformin. (C) In hypoxia, the cytotoxicity of cisplatin was increased by $1 \mathrm{mM}$ metformin. (D and E) Combination treatment increased apoptosis compared to treatment with cisplatin alone in hypoxic conditions. The results represent the mean $\pm \mathrm{SE}$ of three independent experiments; ${ }^{*} \mathrm{P}<0.05$ and ${ }^{* *} \mathrm{P}<0.01$ vs. cisplatin alone, as determined by Student's t-test or Welch's t-test. CDDP, cisplatin; ANOVA, one-way analysis of variance.

in agreement with the results in Fig. 5, metformin may mediate resistance to cisplatin in hypoxic conditions through HIF-1 $\alpha$.

\section{Discussion}

In the present study, we examined the potential of the combination of low-dose metformin and cisplatin to induce apoptosis in hypoxic HepG2 cells. Our results revealed that metformin enhances the antitumor effect of cisplatin in hypoxic conditions in this cell line. Although cisplatin has an antitumor effect in normoxia, its effect was greatly attenuated in hypoxia. Low-dose metformin $(1 \mathrm{mM})$ did not induce cytotoxicity in hypoxic HepG2 cells, and enhanced apoptosis induced by cisplatin but only in hypoxic conditions. This study is the first report to reveal a synergistic effect of metformin and cisplatin on hypoxic HepG2 cells.

Tumor cells show resistance to treatment in hypoxia. The transcription factor HIF-1 $\alpha$ accumulates in tumor cells in hypoxic conditions and is involved in the acquired resistance to cancer therapy and adaptation to hypoxia. HIF-1 $\alpha$ is transported into the nucleus and promotes the expression of many genes involved in angiogenesis, cell proliferation, glucose metabolism, and apoptosis. Furthermore, intracellular accumulation of HIF-1 $\alpha$ inhibits the production of ROS induced by hypoxic stress, and HIF-1 $\alpha$ plays an important role in the cell's adaptation to hypoxia (23). Furthermore, tumor cells undergo epithelial-mesenchymal transition (EMT) in a hypoxic environment, increasing the possibility of invasiveness and metastasis. HIF-1 $\alpha$ activation is potentially involved in the process of EMT induced by hypoxia (9). With this in mind, hypoxia-induced HIF-1 $\alpha$ is considered a therapeutic target to overcome chemotherapy resistance, and various HIF-1 inhibitors are being studied (23-26).

A previous report has indicated that metformin may improve treatment resistance in hypoxic cell lines (27). Metformin is widely used worldwide as a therapeutic agent for type 2 diabetes, but also has antitumor effects in some types of cancer cell lines, including cholangiocarcinoma, 
A

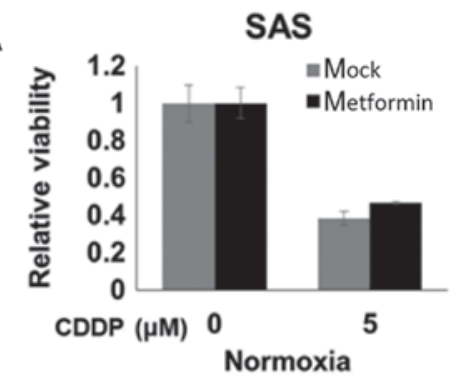

C

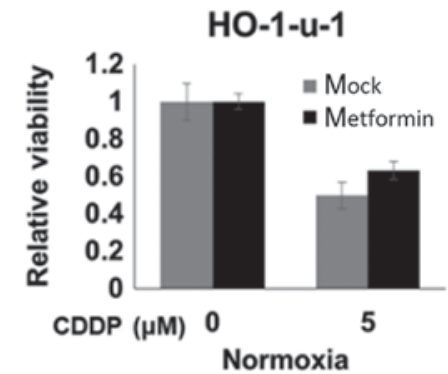

E

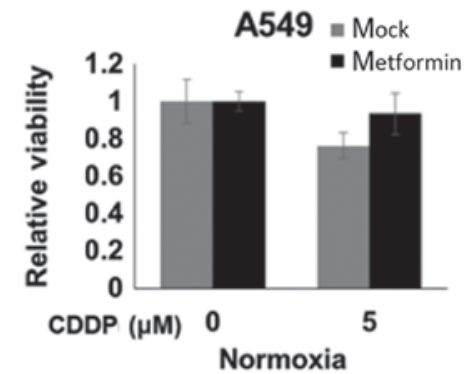

G
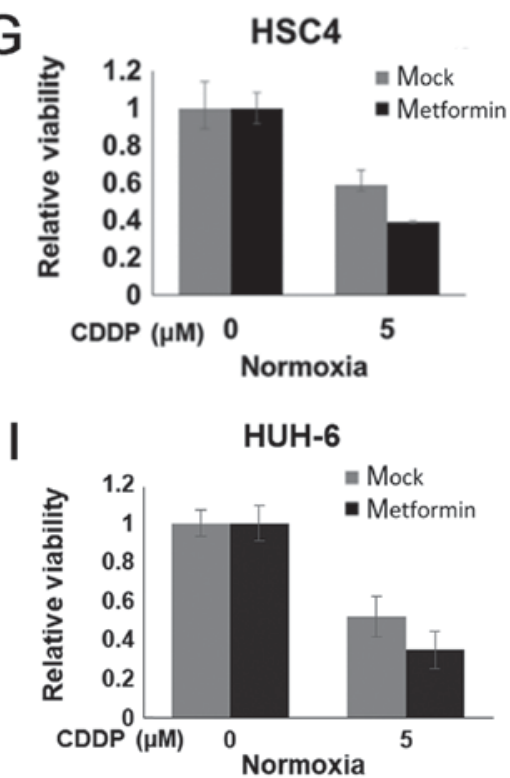

B

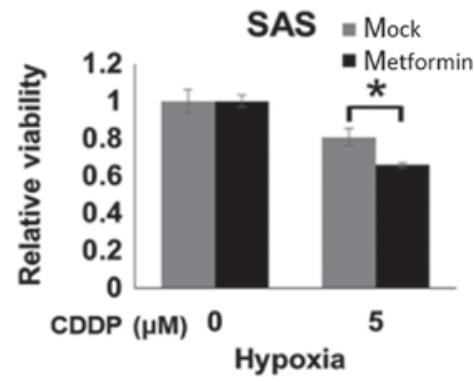

D

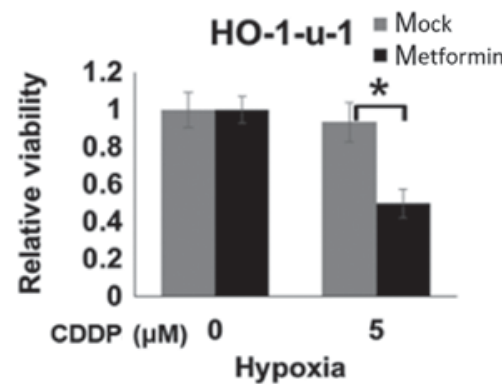

F

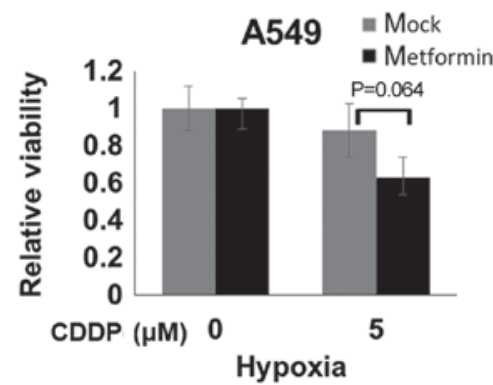

$\mathrm{H}$

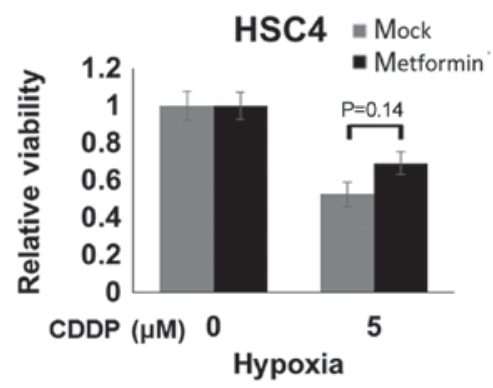

J

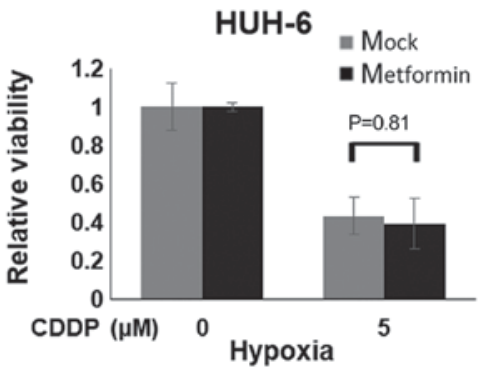

Figure 5. Metformin exhibited a similar effect of reducing treatment resistance in hypoxia in other cells. (A-D) The cytotoxic effect of cisplatin in hypoxia was attenuated in SAS and Ho-1-u-1 cells. Combination treatment significantly reduced viable cells in hypoxia (SAS; P=0.04, HO-1-u-1; P=0.03). (E-J) No significant change was seen with HSC4 cells, A549 and HuH-6 cells. The results represent the mean \pm SE of three independent experiments; ${ }^{*} \mathrm{P}<0.05$ mock vs. metformin, as determined by Student's t-test or Welch's t-test. CDDP, cisplatin; Met, metformin.

ovarian cancer, pancreatic cancer, and epidermoid carcinoma (28-31). Metformin exhibits its antitumor effects via inhibition of mTOR by activation of AMPK (32). A recent report showed that metformin suppresses the above-mentioned HIF-1 expression (27). In our study, metformin did not activate AMPK, but inhibition of Akt,
mTOR, and HIF-1 $\alpha$ was observed. Metformin affects proteins other than AMPK. Metformin suppresses the phosphoinositide 3-kinase/Akt/mTOR pathway through inhibiting the action of insulin-like growth factor-1 (33). Also, Metformin can affect the same pathway suppressively via inhibition of Rag GTPase-mediated mTOR complex 

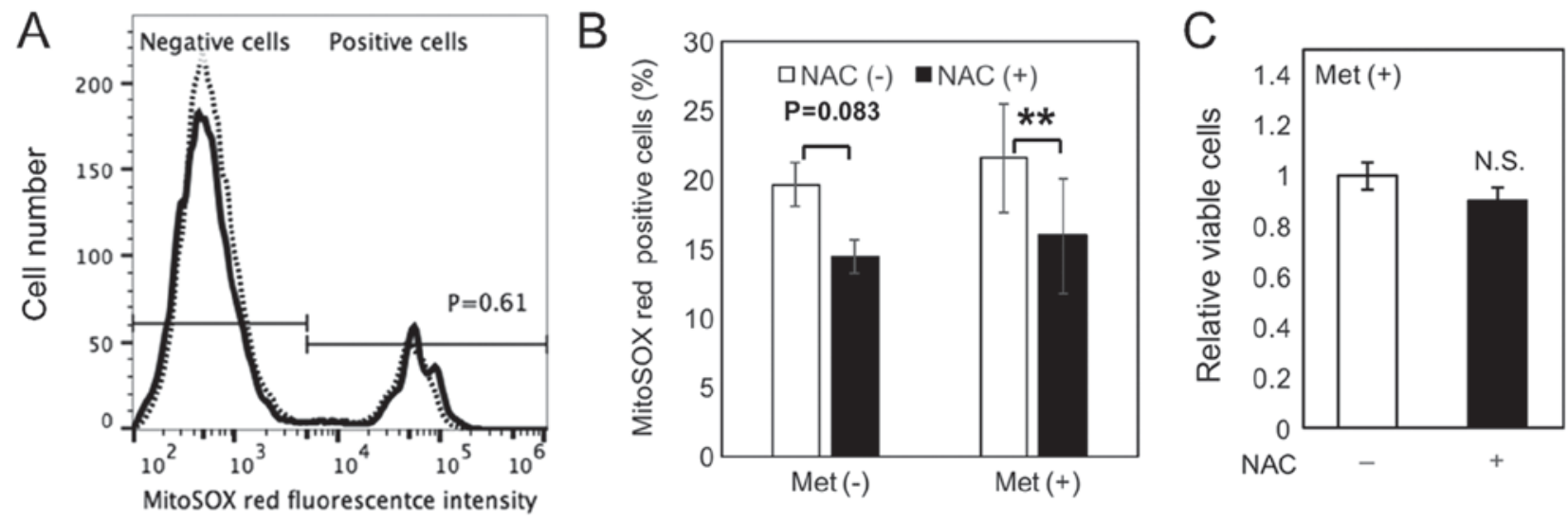

Figure 6. ROS was not involved in metformin-mediated reduction in treatment resistance. (A) The solid line represents the cisplatin only group (19.7 $\pm 1.6 \%$ ), and the dashed line shows the cisplatin and metformin group (21.6 $\pm 3.9 \%)$. No significant difference was seen in MitoSOX Red-positive cells between these two groups $(\mathrm{P}=0.61)$. (B) NAC $(10 \mathrm{mM})$ induced a decrease in MitoSOX RED-positive cells in both mock and metformin-treated cells $(14.5 \pm 1.2,15.9 \pm 4.1 \%$, respectively). ${ }^{* *} \mathrm{P}<0.01$ vs. with NAC, as determined by Student's t-test. (C) NAC did not reduce the number of viable cells. The results represent the mean \pm SE of three independent experiments. N.S., no significant difference (P>0.05). ROS, reactive oxygen species; Met, metformin; NAC, N-acetyl-1-cysteine.
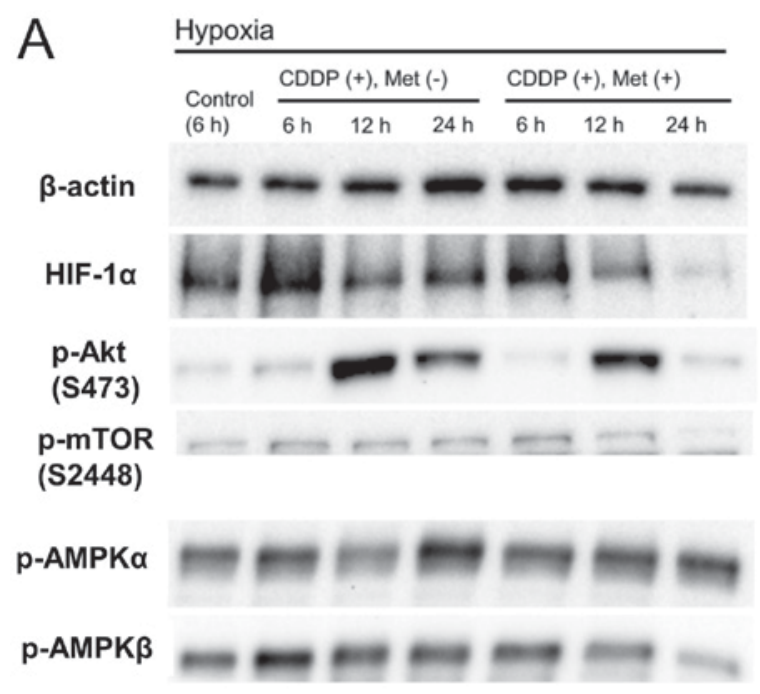

$\mathrm{B}$

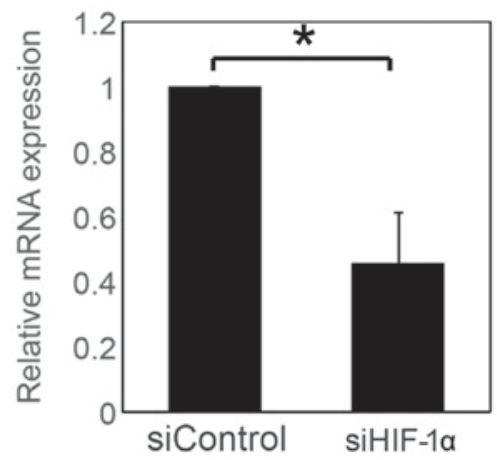

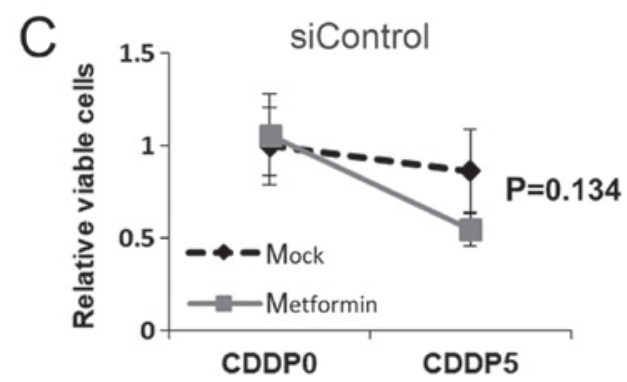

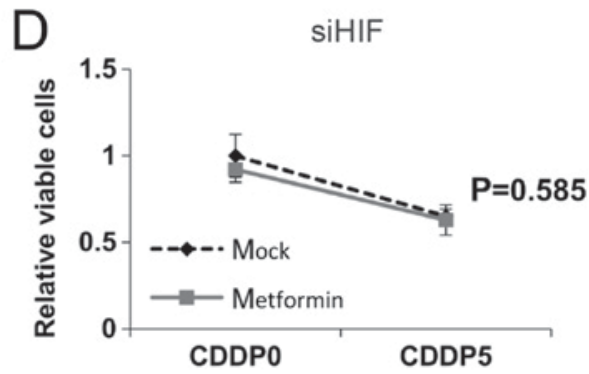

Figure 7. Hypoxia-induced factor $1 \alpha$ mediates a pathway in which metformin modulates hypoxia-induced resistance to cisplatin. (A) HepG2 cells expressed HIF-1 $\alpha$ in hypoxic conditions in the absence of metformin. Metformin suppressed the expression of HIF-1 $\alpha$. Metformin did not activate AMPK $\alpha$ or AMPK $\beta$, but activated Akt and mTOR. This change was remarkable at $24 \mathrm{~h}$. (B) siRNA significantly inhibited HIF-1 $\alpha$ gene expression. The effect of combining metformin were less modulated in siHIF-treated cells. (C) sicontrol: $\mathrm{P}=0.134$, (D) siHIF; $\mathrm{P}=0.585$; ${ }^{\mathrm{P}}<0.05$. CDDP, cisplatin; HIF, hypoxia-inducible factor; AMPK, adenosine monophosphate-activated protein kinase; p-, phosphorylated; si, small interfering; mTOR, mammalian target of rapamycin.

1 signaling (34). Promotion of the degradation of HIF-1 $\alpha$ by suppression of its stability is one mechanism of action of metformin (35). These mechanisms are thought to be involved in improving hypoxia-induced cisplatin resistance. Metformin inhibits complex I of the mitochondrial respiratory chain, an important source of ROS production (36). In our study, metformin did not induce production of ROS, and ROS was not involved in the effect of a low concentration of metformin. A possible effect of metformin on ROS may be concentration dependent. Our results also confirmed that by knocking down the HIF-1 $\alpha$ gene, hypoxia-induced resistance to cisplatin disappeared in HepG2 cells, and the 
synergistic effect of cisplatin and metformin disappeared as well. The effect of a low concentration of metformin was confirmed by hypoxia-induced resistance that was mediated by HIF-1 $\alpha$. In addition, we also confirmed the effect of combining metformin on other cells that acquired cisplatin resistance in hypoxic conditions. These results suggest that metformin may improve the treatment efficacy by selectively affecting on the resistant fraction to cisplatin included in the heterogeneous tumor tissue in multiple cancer types. Metformin is a safe drug compared to other biguanides, but lactic acidosis is a serious side effect (37). With oral administration, obtaining a blood concentration that is needed to affect tumors will increase the amount of medicine to be administered, and side effects are likely to result. Therefore, effective treatment at low doses is clinically necessary.

For many high-risk hepatoblastoma cases, surgical resection as a definitive therapy is difficult, and neoadjuvant chemotherapy is often performed to reduce the tumor size before surgery. However, this approach is often ineffective, and recently the efficacy of TACE has attracted increasing attention (7,38-40). TACE can deliver anticancer drugs to tumors at high concentrations, potentially increasing the likelihood of a robust antitumor response. TACE may be particularly applicable for hepatoblastoma (38). Hirakawa et al (7) reported that by adding TACE to systemic chemotherapy, the mean tumor reduction rate improved from 44.3 to $60.9 \%$ and the mean $\alpha$ fetoprotein reduction rate improved from 63.4 to $94.8 \%$. Performing TACE before surgery improves bleeding control and reduces the tumor mass, resulting in improved resection efficacy (39). TACE has been suggested as a treatment option for chemoresistant unresectable cases for which liver transplantation is not an option or as 'bridging' treatment for those patients awaiting liver transplantation (40). However, the TACE procedure may increase the risk of resistance to chemotherapy by occluding the blood flow and inducing hypoxia with a concomitant risk of invasion and recurrence through EMT due to changes in the tumor microenvironment (41). Our current results suggested that low-dose metformin $(1 \mathrm{mM})$ may reduce resistance to cisplatin in hypoxic conditions that result from TACE. Therefore, administration of metformin in combination with TACE for hepatoblastoma may have very practical advantages for improving treatment intensity and reducing recurrence. Further in vivo studies are needed in which cisplatin and metformin are administered by catheter placement into the hepatic artery of animal models.

In conclusion, we have shown that a low concentration of metformin attenuates hypoxia-induced resistance to cisplatin in HepG2 cells. Thus, low-dose metformin has high potential to enhance the therapeutic effect of cisplatin in hepatoblastoma and to improve the prognosis of patients.

\section{Acknowledgements}

Not applicable.

\section{Funding}

The present study was supported by JSPS KAKENHI grant no. JP17K10430.

\section{Availability of data and materials}

The datasets used and/or analyzed during the current study are available from the corresponding author on reasonable request.

\section{Authors' contributions}

MA and YT supervised the entire study, participated in study design and coordination. $\mathrm{HF}, \mathrm{KH}$, and MS performed the experiments and statistical analyses. TF and IF helped the experiments. $\mathrm{HF}$ drafted the manuscript and $\mathrm{KH}$ revised this manuscript. All authors read and approved the final manuscript.

\section{Ethics approval and consent to participate}

Not applicable.

\section{Patient consent for publication}

Not applicable.

\section{Competing interests}

The authors declare that they have no competing interests.

\section{References}

1. Hiyama E: Pediatric hepatoblastoma: Diagnosis and treatment. Transl Pediatr 3: 293-299, 2014.

2. Czauderna P, Otte JB, Roebuck DJ, von Schweinitz D and Plaschkes J: Surgical treatment of hepatoblastoma in children. Pediatr Radiol 36: 187-191, 2006.

3. Aronson DC, Czauderna P, Maibach R, Perilongo G and Morland B: The treatment of hepatoblastoma: Its evolution and the current status as per the SIOPEL trials. J Indian Assoc Pediatr Surg 19: 201-207, 2014.

4. Zsiros J, Brugieres L, Brock P, Roebuck D, Maibach R, Zimmermann A, Childs M, Pariente D, Laithier V, Otte JB, et al: Dose-dense cisplatin-based chemotherapy and surgery for children with high-risk hepatoblastoma (SIOPEL-4): A prospective, single-arm, feasibility study. Lancet Oncol 14: 834-842, 2013.

5. Ma J, Gimenez JM, Sandow T, Devun D, Kirsch D, Gulotta P, Gilbert P and Kay D: Intraarterial liver-directed therapies: The role of interventional oncology. Ochsner J 17: 412-416, 2017.

6. Hishiki T: Current therapeutic strategies for childhood hepatic tumors: Surgical and interventional treatments for hepatoblastoma. Int J Clin Oncol 18: 962-968, 2013.

7. Hirakawa M, Nishie A, Asayama Y, Fujita N, Ishigami K, Tajiri T, Taguchi T and Honda H: Efficacy of preoperative transcatheter arterial chemoembolization combined with systemic chemotherapy for treatment of unresectable hepatoblastoma in children. Jpn J Radiol 32: 529-536, 2014.

8. Miyayama S and Matsui O: Superselective conventional transarterial chemoembolization for hepatocellular carcinoma: Rationale, technique, and outcome. J Vasc Interv Radiol 27: 1269-1278, 2016.

9. Sato M, Hirose K, Aoki M, Hatayama Y, Kawaguchi H, Akimoto H, Narita Y and Takai Y: How can we overcome hypoxia- and radiation-induced EMT? Contribution of blockade on HIF-1 and JNK phospholyration with LW6. Int J Radiat Oncol 90: S771, 2014.

10. Zakikhani M, Dowling R, Fantus IG, Sonenberg N and Pollak M: Metformin is an AMP kinase-dependent growth inhibitor for breast cancer cells. Cancer Res 66: 10269-10273, 2006.

11. Alimova IN, Liu B, Fan Z, Edgerton SM, Dillon T, Lind SE and Thor AD: Metformin inhibits breast cancer cell growth, colony formation and induces cell cycle arrest in vitro. Cell Cycle 8: 909-915, 2009. 
12. Cantrell LA, Zhou C, Mendivil A, Malloy KM, Gehrig PA and Bae-Jump VL: Metformin is a potent inhibitor of endometrial cancer cell proliferation-implications for a novel treatment strategy. Gynecol Oncol 116: 92-98, 2010.

13. Ben Sahra I, Laurent K, Giuliano S, Larbret F, Ponzio G, Gounon P, Le Marchand-Brustel Y, Giorgetti-Peraldi S, Cormont M, Bertolotto $\mathrm{C}$, et al: Targeting cancer cell metabolism: The combination of metformin and 2-deoxyglucose induces p53-dependent apoptosis in prostate cancer cells. Cancer Res 70 2465-2475, 2010.

14. Rattan R, Graham RP, Maguire JL, Giri S and Shridhar V: Metformin suppresses ovarian cancer growth and metastasis with enhancement of cisplatin cytotoxicity in vivo. Neoplasia 13 : 483-491, 2011.

15. Bao B, Wang Z, Ali S, Ahmad A, Azmi AS, Sarkar SH, Banerjee S, Kong D, Li Y, Thakur S and Sarkar FH: Metformin inhibits cell proliferation, migration and invasion by attenuating CSC function mediated by deregulating miRNAs in pancreatic cancer cells. Cancer Prev Res (Phila) 5: 355-364, 2012.

16. Kato K, Gong J, Iwama H, Kitanaka A, Tani J, Miyoshi H, Nomura K, Mimura S, Kobayashi M, Aritomo Y, et al: The antidiabetic drug metformin inhibits gastric cancer cell proliferation in vitro and in vivo. Mol Cancer Ther 11: 549-560, 2012.

17. Storozhuk Y, Hopmans SN, Sanli T, Barron C, Tsiani E, Cutz JC, Pond G, Wright J, Singh G and Tsakiridis T: Metformin inhibits growth and enhances radiation response of non-small cell lung cancer (NSCLC) through ATM and AMPK. Br J Cancer 108: 2021-2032, 2013.

18. Hirsch HA, Iliopoulos D, Tsichlis PN and Struhl K: Metformin selectively targets cancer stem cells, and acts together with chemotherapy to block tumor growth and prolong remission. Cancer Res 69: 7507-7511, 2009.

19. Song CW, Lee H, Dings RP, Williams B, Powers J, Santos TD, Choi $\mathrm{BH}$ and Park HJ: Metformin kills and radiosensitizes cancer cells and preferentially kills cancer stem cells. Sci Rep 2: $362,2012$.

20. Cai X, Hu X, Cai B, Wang Q, Li Y, Tan X, Hu H, Chen X, Huang J, Cheng J and Jing X: Metformin suppresses hepatocellular carcinoma cell growth through induction of cell cycle G1/G0 phase arrest and p21CIP and p27KIP expression and downregulation of cyclin D1 in vitro and in vivo. Oncol Rep 30: 2449-2457, 2013.

21. Zhang M, Moore GA, Lever M, Gardiner SJ, Kirkpatrick CM and Begg EJ: Rapid and simple high-performance liquid chromatographic assay for the determination of metformin in human plasma and breast milk. J Chromatogr B Analyt Technol Biomed Life Sci 766: 175-179, 2002.

22. Mogavero A, Maiorana MV, Zanutto S, Varinelli L, Bozzi F, Belfiore A, Volpi CC, Gloghini A, Pierotti MA and Gariboldi M: Metformin transiently inhibits colorectal cancer cell proliferation as a result of either AMPK activation or increased ROS production. Sci Rep 7: 15992, 2017.

23. Sato M, Hirose K, Kashiwakura I, Aoki M, Kawaguchi H, Hatayama Y, Akimoto H, Narita Y and Takai Y: LW6, a hypoxia-inducible factor 1 inhibitor, selectively induces apoptosis in hypoxic cells through depolarization of mitochondria in A549 human lung cancer cells. Mol Med Rep 12: 3462-3468, 2015.

24. Carroll CE, Liang Y, Benakanakere I, Besch-Williford C and Hyder SM: The anticancer agent YC-1 suppresses progestin-stimulated VEGF in breast cancer cells and arrests breast tumor development. Int J Oncol 42: 179-187, 2013.

25. Kong D, Park EJ, Stephen AG, Calvani M, Cardellina JH, Monks A, Fisher RJ, Shoemaker RH and Melillo G: Echinomycin, a small-molecule inhibitor of hypoxia-inducible factor-1 DNA-binding activity. Cancer Res 65: 9047-9055, 2005.
26. Chen J, Wang J, Schwab LP, Park KT, Seagroves TN, Jennings LK, Miller DD and Li W: Benzimidazole analogs as potent hypoxia inducible factor inhibitors: Synthesis, biological evaluation, and profiling drug-like properties. Anticancer Res 34: 3891-3904, 2014

27. Guimarães TA, Farias LC, Santos ES, de Carvalho Fraga CA, Orsini LA, de Freitas Teles L, Feltenberger JD, de Jesus SF, de Souza MG, Santos SH, et al: Metformin increases PDH and suppresses HIF-1 $\alpha$ under hypoxic conditions and induces cell death in oral squamous cell carcinoma. Oncotarget 7: 55057-55068, 2016.

28. Fujimori T, Kato K, Fujihara S, Iwama H, Yamashita T, Kobayashi K, Kamada H, Morishita A, Kobara H, Mori H, et al: Antitumor effect of metformin on cholangiocarcinoma: In vitro and in vivo studies. Oncol Rep 34: 2987-2996, 2015.

29. Wu B, Li S, Sheng L, Zhu J, Gu L, Shen H, La D, Hambly BD, Bao S and Di W: Metformin inhibits the development and metastasis of ovarian cancer. Oncol Rep 28: 903-908, 2012.

30. Kato K, Iwama H, Yamashita T, Kobayashi K, Fujihara S, Fujimori T, Kamada H, Kobara H and Masaki T: The anti-diabetic drug metformin inhibits pancreatic cancer cell proliferation in vitro and in vivo: Study of the microRNAs associated with the antitumor effect of metformin. Oncol Rep 35: 1582-1592, 2016.

31. Liu Y, Zhang Y, Jia K, Dong Y and Ma W: Metformin inhibits the proliferation of A431 cells by modulating the PI3K/Akt signaling pathway. Exp Ther Med 9: 1401-1406, 2015.

32. Rocha GZ, Dias MM, Ropelle ER, Osório-Costa F, Rossato FA, Vercesi AE, Saad MJ and Carvalheira JB: Metformin amplifies chemotherapy-induced AMPK activation and antitumoral growth. Clin Cancer Res 17: 3993-4005, 2011.

33. Karnevi E, Said K, Andersson R and Rosendahl AH: Metformin-mediated growth inhibition involves suppression of the IGF-I receptor signalling pathway in human pancreatic cancer cells. BMC Cancer 13: 235, 2013

34. Kalender A, Selvaraj A, Kim SY, Gulati P, Brûlé S, Viollet B, Kemp BE, Bardeesy N, Dennis P, Schlager JJ, et al: Metformin, independent of AMPK, inhibits mTORC1 in a rag GTPase-dependent manner. Cell Metab 11: 390-401, 2010.

35. Zhou X, Chen J, Yi G, Deng M, Liu H, Liang M, Shi B, Fu X, Chen Y, Chen L, et al: Metformin suppresses hypoxia-induced stabilization of HIF-1 $\alpha$ through reprogramming of oxygen metabolism in hepatocellular carcinoma. Oncotarget 7: 873-884, 2016.

36. Bridges HR, Jones AJ, Pollak MN and Hirst J: Effects of metformin and other biguanides on oxidative phosphorylation in mitochondria. Biochem J 462: 475-487, 2014

37. Yokoyama S, Tsuji H, Hiraoka S and Nishihara M: Investigation of risk factors affecting lactate levels in Japanese patients treated with metformin. Biol Pharm Bull 39: 2022-2027, 2016.

38. Zhang J, Xu F, Chen K, Zhou S, Li H, Niu C and Tan X: An effective approach for treating unresectable hepatoblastoma in infants and children: Pre-operative transcatheter arterial chemoembolization. Oncol Lett 6: 850-854, 2013.

39. Vogl TJ, Scheller A, Jakob U, Zangos S, Ahmed M and Nabil M: Transarterial chemoembolization in the treatment of hepatoblastoma in children. Eur Radiol 16: 1393-1396, 2006.

40. Czauderna P, Zbrzezniak G, Narozanski W, Korzon M, Wyszomirska M and Stoba C: Preliminary experience with arterial chemoembolization for hepatoblastoma and hepatocellular carcinoma in children. Pediatr Blood Cancer 46: 825-828, 2006.

41. Rhee H, Nahm JH, Kim H, Choi GH, Yoo JE, Lee HS, Koh MJ and Park YN: Poor outcome of hepatocellular carcinoma with stemness marker under hypoxia: Resistance to transarterial chemoembolization. Mod Pathol 29: 1038-1049, 2016. 